\title{
A rare case of bilateral antero-internal shoulder dislocation in a judo player: a case study and review of the literature
}

\author{
Omar Fadili, Mohamed Laffani, Souhail Echoual, Abdellah Chrak, Bienvenu Jean Celien Okouango, \\ Mustapha Fadili \\ Department of Traumatology-Orthopedic and Reconstructive Surgery, Ibn Rochd University Hospital, Casablanca, Morocco
}

\begin{abstract}
Pure anterior bilateral shoulder dislocations are rare clinical features, especially in traumatic forms. They are most often posterior, occurring during an epileptic seizure. Few cases are described in the literature, and the mechanism varies from case to case. We report a specific case of pure bilateral anterior shoulder dislocation in a 29 -year-old judo player following an accident during his training and discuss the circumstances, mechanism, treatment, and prognosis.
\end{abstract}

Keywords: Shoulder dislocation; Glenohumeral dislocation; Bilateral dislocation; Judo; Sport

Glenohumeral dislocations are the most frequent of all dislocations. The bilateral form is rare, dominated by the posterior variant that often occurs as part of the "triple E syndrome" secondary to epileptic seizures causing violent and synchronous muscle contractions of the two shoulders, pushing back the humeral head most often in the posterior direction [1]. We report a rare case of pure anterior dislocation following a particular mechanism in a 29 -year-old judo player and discuss the circumstances, radiological appearance, treatment, and prognosis with a literature review.

\section{CASE REPORT}

In April 2021, a 29-year-old a judo player with no notable pathological history (no history of previous dislocation, join laxity, head trauma, or epilepsy) consulted in the emergency room for blunt trauma to both shoulders that occurred during a training session. As he lifted his 70-kg opponent, he tipped backwards, feeling dislocation in both shoulders and evidenced by a mechanism of protraction, abduction, and forced pronation. The patient presented with severe pain and absolute functional impairment. Clinical examination found typical signs of anterior dislocations of the shoulder on both sides; sensitivity in the territory of the axillary nerve was preserved, and the radial pulse was felt in both limbs. X-ray assessment (Anteroposterior and lateral views) confirmed the diagnosis of bilateral antero-internal sub-coracoid dislocation (Figs. 1 and 2). The two dislocations were reduced by the Milch technique under general anesthesia, and both shoulders were immobilized in adduction and internal rotation by an orthosis. A follow-up X-ray was performed to appreciate the reduction of both dislocations (Fig. 3). At 3 weeks, the immobilization restraint was removed, and rehabilitation be-

Received: May 6, $2021 \quad$ Revised: May 27, $2021 \quad$ Accepted: June 1, 2021

Correspondence to: Omar Fadili

Department of Traumatology-Orthopedic and Reconstructive Surgery, Ibn Rochd University Hospital, Casablanca 20000, Morocco

Tel: +212-64-818-2666, E-mail: drfadiliomar@outlook.fr, ORCID: https://orcid.org/0000-0003-4577-9997

Financial support: None.

Conflict of interest: None.

Copyright@ 2021 Korean Shoulder and Elbow Society.

This is an Open Access article distributed under the terms of the Creative Commons Attribution Non-Commercial License (http://creativecommons.org/licenses/by-nc/4.0/) which permits unrestricted non-commercial use, distribution, and reproduction in any medium, provided the original work is properly cited. 


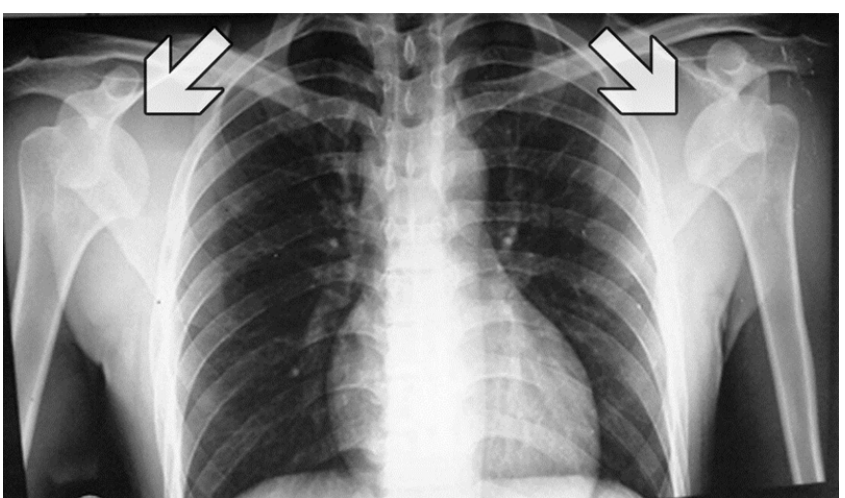

Fig. 1. Bilateral antero-internal dislocation of the shoulders: sub-coracoid variety (front view). Internal deplacement indicated by the arrows.
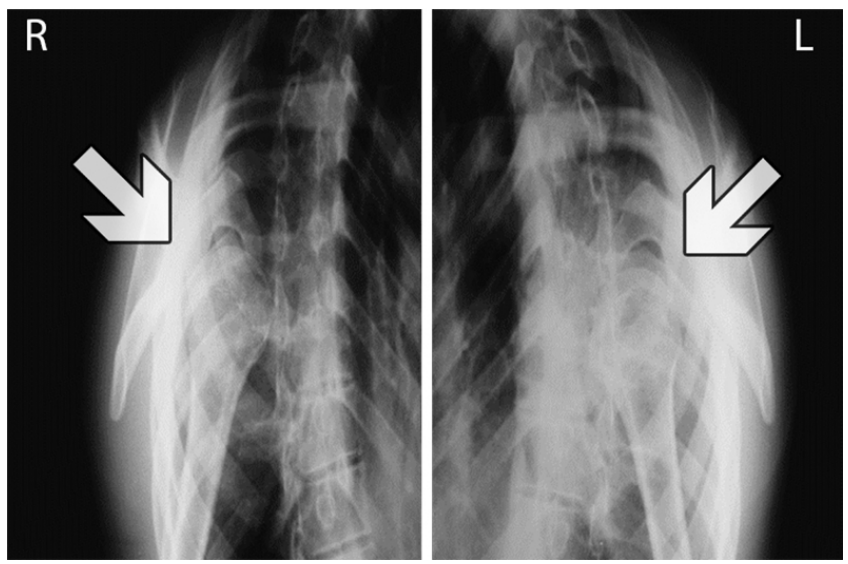

Fig. 2. Antero-internal dislocation of both shoulders (lateral views). Anterior deplacement indicated by the arrows.

gan.

The duration of the rehabilitation protocol was 6 weeks, divided into three stages ( 2 weeks each). Stage 1 was to control the pain and inflammation caused by the dislocation: external and internal rotation exercises (it is important to avoid positions that could cause repeat dislocation); stage 2 was to restore muscle strength and range of motion: deltoid muscle, trapezius muscle, and serratus anterior muscle. Exercises in this stage were performed widely in the horizontal and diagonal axes; and stage 3 was designed for endurance, plyometric, and strength exercises as well as extremity weight rehabilitation exercises.

At 9 weeks after the accident, both shoulders had satisfactory mobility: abduction was $160^{\circ}$, internal rotation reached D4, and external rotation was $35^{\circ}$ to the right and $40^{\circ}$ to the left. The tenderness and contractility of the stumps of both shoulders were normal. There were no signs of abnormal joint laxity. The patient resumed training at 12 weeks. At the last follow-up at 18 months, shoulder mobility was normal, and there was no instability or re-
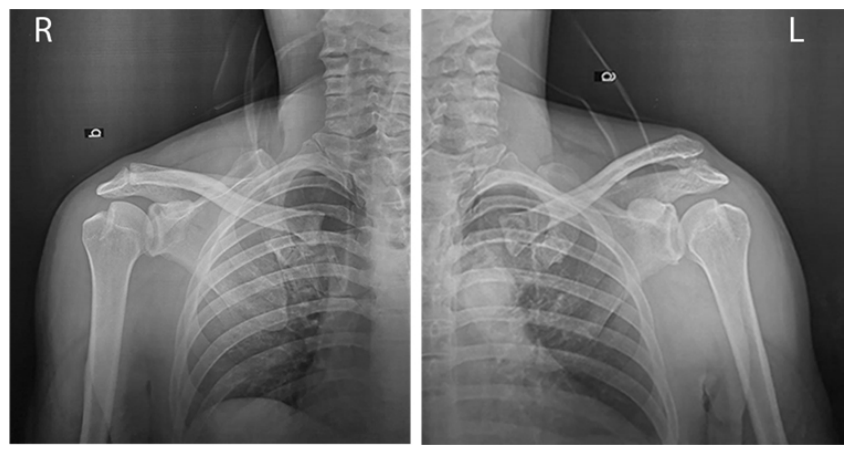

Fig. 3. Follow-up X-ray showing reduction of both dislocations.

currence.

\section{DISCUSSION}

Shoulder dislocation is recognized as the most common dislocation in the body ( $85 \%$ of cases). It is anterior in $98 \%$ of cases. However, bilateral shoulder dislocation is rare and uncommon. The first case was described in 1902 following excessive muscle contractions caused by camphor overdose [2]. The condition has been the subject of few publications [3,4]. Brown [5] in 1984 reported a series of 90 cases of bilateral dislocations and described three etiologies: violent muscle contractions (49\%), direct trauma (23\%), and absence of trauma (36\%). These dislocations are most frequently posterior [6]; described under the name of "triple E syndrome" (epilepsy or any seizure, electrocution, and extreme trauma) [1]. The anterior variety is rare, with only 30 reported cases [6], representing $0.5 \%$ of bilateral shoulder dislocations [7-9].

Indeed, the force necessary to produce these dislocations must act symmetrically and synchronously at the level of the two glenohumeral joints. This most often occurs with a seizure or neuromuscular disease [3-5]. Bouras et al. [6] described a case of bilateral anterior shoulder dislocation in an 18-year-old bodybuilder who was lifting a straight $40-\mathrm{kg}$ bar that fell backward, causing dislocation. The etiologies of the anterior bilateral dislocations reported are all post-traumatic, while those of posterior dislocations are non-traumatic and often due to excessive muscle contractions. Three other cases have been reported in patients with bilateral shoulder dislocation occurring during sports training (bodybuilding) without history of instability and who lifted bars during a "bench-press" weight training session [4-7]. The associated mechanism was hyperextension, abduction, and forced rotation of both shoulders while all pivot movement was blocked by the bench, which simultaneously pushed the two humeral heads forward under the weight of the load. This mechanism also can occur when performing a judo technique called morote gari, 
which consists of a frontal attack characterized by a double leg takedown. It is accomplished by wrapping both arms around the opponent's legs and by pushing one's shoulder against their stomach to push them backward for a throw.

Reduction of the dislocation was routine under general anesthesia by Milch method. We did not want to reduce the injuries under sedation, a method usually used in the department for dislocated shoulder, believing that the pain could cause a reflex contracture that could prevent reduction of one or two dislocations. The orthopedic treatment provided resulted in a good outcome. This is reassuring regarding therapeutic management, with surgery being considered only in the event of recurrence, which is more common in patients younger than 40 years [10].

Bilateral shoulder dislocation is a rare injury, and a post-traumatic etiology is exceptional. We report an unusual occurrence of the causal mechanism.

\section{ORCID}

Omar Fadili https://orcid.org/0000-0003-4577-9997

\section{REFERENCES}

1. Betz ME, Traub SJ. Bilateral posterior shoulder dislocations following seizure. Intern Emerg Med 2007;2:63-5.

2. Mynter H. XIV. Subacromial dislocation from muscular spasm. Ann Surg 1902;36:117-9.

3. Dunlop CC. Bilateral anterior shoulder dislocation: a case report and review of the literature. Acta Orthop Belg 2002;68:16870.

4. Koufagued K, Chafry B, Bouabid S, Chagar B. Pure bilateral anterior dislocations of the shoulder of special mechanism: report of two cases. Pan Afr Med J 2015;22:178.

5. Brown RJ. Bilateral dislocation of the shoulders. Injury 1984; 15:267-73.

6. Bouras Y, Elandaloussi Y, Nadil MA, et al. Bilateral anterior dislocation of shoulders (a case report). J Traumatol Sport 2009;26: 247-9.

7. Cresswell TR, Smith RB. Bilateral anterior shoulder dislocations in bench pressing: an unusual cause. Br J Sports Med 1998;32: 71-2.

8. Singh S, Kumar S. Bilateral anterior shoulder dislocation: a case report. Eur J Emerg Med 2005;12:33-5.

9. Petty K, Price J, Kharasch M, Novack J. Bilateral luxatio erecta: a case report. J Emerg Med 2014;46:176-9.

10. El Andaloussi Y, Arssi M, Zaouari T, et al. Anterior shoulder instability: review of 36 athletic shoulders. J Traumatol Sport 2006;23:148-52. 